\title{
Research on Recognition Method of COVID-19 Images Based on Deep Learning
}

\author{
Dongsheng $\mathrm{Ji}^{1, *}$, Yanzhong Zhao ${ }^{1}$, Zhujun Zhang ${ }^{1}$, and Qianchuan Zhao ${ }^{12}$ \\ ${ }^{1}$ School of Computer and Communication, Lanzhou University of Technology, LanZhou, 730050 \\ ${ }^{2}$ Intelligence and Network Information Center, Tsinghua University, Beijing, 100084
}

\begin{abstract}
In view of the large demand for new coronary pneumonia covid19 image recognition samples, the recognition accuracy is not ideal. In this paper, a new coronary pneumonia positive image recognition method proposed based on small sample recognition. First, the CT image pictures are preprocessed, and the pictures are converted into the picture formats which are required for transfer learning. Secondly, small-sample image enhancement and extension are performed on the transformed image, such as staggered transformation, random rotation and translation, etc.. Then, multiple migration models are used to extract features and then perform feature fusion. Finally, the model is adjusted by fine-tuning. Then train the model to obtain experimental results. The experimental results show that our method has excellent recognition performance in the recognition of new coronary pneumonia images, even with only a small number of CT image samples.
\end{abstract}

\section{Introduction}

As of July 30,2020 , there have been $17,039,160$ people with new coronary pneumonia in the world, and it is growing at a rate of close to 300,000 per day [1]. In the diagnosis and treatment plan of the "New Coronavirus Infection Pneumonia Diagnosis and Treatment Plan (Trial Seventh Edition)" issued by the National Health and Medical Commission, in addition to the incubation period 1-14 days isolation observation based on epidemiological investigations, pneumonia symptoms based on clinical manifestations and the characteristics of the signs of illness, the results of laboratory nasopharyngeal swabs and nucleic acid testing of Yin/Yang, and effective oxygen therapy combined with antiviral and antimicrobial therapy, the most important diagnostic criterion is chest imaging. Based on the accumulation of clinical experience in the fight against new coronary pneumonia and the in-depth related research, some false negatives in the new coronavirus nucleic acid test may be questioned, especially for those with a negative nucleic acid test and typical CT findings, which may affect clinical investigation and treatment [2]. On February 4, 2020, the National Health Commission released the latest "New Coronavirus Pneumonia Diagnosis and Treatment Plan" (Fifth Edition) to update the fourth version. While confirming the diagnosis and paying attention to the imaging findings, the patients $\mathrm{CT}$ imaging findings are also included in the discharge criteria, and imaging findings are used as the basis for clinical suspicion and diagnosis in Hubei [3]. Compared with images of other modalities, CT images have higher definition and resolution. Therefore, chest imaging examination is an important medical imaging method for timely identification of COVID-19 cases. Through the diagnosis of chest imaging, medical staff can grasp the imaging characteristics of COVID-19 cases more accurately, such as early appearance of multiple small patchy shadows and interstitial changes, which are obvious outside the lung. Then, it develops into multiple ground-glass shadows and infiltration shadows in both lungs. In severe cases, lung consolidation and pleural effusion are rare. It has important guiding value for accurately estimating the condition and its development, formulating treatment plans and evaluating prognosis.

This article uses the domain knowledge obtained from the detection and recognition of medical imaging targets to assist doctors in the diagnosis and treatment of COVID-19 disease, which is conducive to the early detection of lung lesions, etc., especially telemedicine and diagnosis, which has very important application value and Academic significance. The intervention of the application of computer intelligent image analysis technology provides new ideas and ways to accurately identify and assist medical diagnosis of COVID-19 disease. At the same time, it also has a high reference value for the further study of intelligent imaging-assisted diagnosis technology for human severe viral pneumonia diseases and other newly emerging respiratory syndrome diseases.

\footnotetext{
*Corresponding author: jids@lut.edu.cn
} 


\section{Relevant work}

Covid19's image recognition can be regarded as image recognition. Image recognition mainly relies on pattern recognition, and current machine learning is developed from pattern recognition. Changes in common chest CT ground-glass features of new coronary pneumonia are the most common manifestations of COVID-19 infection. The CT manifestations of pneumonia caused by new coronavirus infection are diverse, with ground-glass shadows and actuality becoming dominant, lacking specificity. Generally, the clinical symptoms are mild and the lung lesions are few, which often brings certain difficulties to the diagnosis. For suspected cases, CT examination finds abnormal manifestations in the lungs, which can assist in early treatment and intervention. However, the diagnosis requires a comprehensive judgment based on epidemiological history, new coronavirus testing and radiological manifestations.

On February 28th, a research team led by Academician Nanshan Zhong (37 experts from the China New Coronary Pneumonia Medical Expert Group, research units include the National Respiratory Disease Clinical Research Center, Wuhan Jinyintan Hospital and other 9 authoritative institutions) in the New England Journal of Medicine (NEJM) published the research results on the "Clinical Features of China's 2019 Novel Coronavirus Disease", [4] mainly related to patient symptoms, confirmed patients, clinical symptoms and infection signs of suspected patients. From the perspective of imaging evaluation, chest X-ray or CT image was analyzed for lesion characteristics. The research team used detailed data to compare and analyze the characteristics of SARS, MERS and seasonal influenza cases, showing that the new coronary pneumonia has different trends. Domestic scholars wrote an article in the "Nature" magazine on the study of the characteristics of new coronary pneumonia, and discussed the clinical characteristics and imaging detection characteristics [5]. Researchers from the National Supercomputing Center of Sun Yat-sen University used the parallel computing deep learning method to study the characteristics of COVID-19 imaging lesions [6]. The training model can locate the main regional features, especially ground glass shadows, and the model can be fast. The research team of Central South University studied the short-term disease progression and clinical characteristics of patients with new coronary pneumonia, [7] carried out characteristic studies on chest CT images of confirmed cases, analyzed the severity of the disease, and made clinical treatment recommendations.

Doctors from the Radiology Department of Shanghai Public Health Clinical Center, together with researchers from the Shanghai United Imaging Research and Development Department and doctoral students of Shanghai University, used deep learning to build an automatic segmentation and quantification system based on deep learning, [8] mainly used image segmentation theory for research The chest $\mathrm{CT}$ infection area and the overall structure of the lungs are annotated for each case using the method of man-machine loop optimization.
Researchers from East China Normal University, the Key Laboratory of Artificial Intelligence of the Ministry of Education, Ryerson University in Canada, and Shanghai Jianglai Data Technology Co., Ltd. used depth cameras and deep learning to study human abnormal breathing patterns, which are clinically significant for 6 types Breathing patterns are classified to help large-scale screening of patients infected with COVID-19 [9]. Researchers from Huazhong University of Science and Technology and Wuhan University of Technology used machine learning models to predict the criticality of patients with severe COVID-19, and identify three main clinical features,assessing the risk of death accurately and quickly, which has important clinical significance [10].

Dr. Shuai Wang from the Cancer Hospital of Tianjin Medical University used deep learning methods to extract COVID-19 image features, established a learning model to analyze positive cases, and provided a theoretical basis for timely and accurate diagnosis of COVID-19 [11]. Experts from the Affiliated Hospital of Huazhong University of Science and Technology used three-dimensional CT to detect new coronary pneumonia, and a three-dimensional neural network based on weakly supervised deep learning to classify positive and negative cases to quickly identify COVID-19 cases [12]. Researchers such as Asmaa and Mohammed from Arthurs University and Birmingham City University [13], in view of the high availability of COVID-19 annotated image data sets, use convolutional neural networks to identify and classify new crown images, and use the class decomposition mechanism to study its class boundary to deal with the irregularities in the data set, and having great performance.

\section{Based on small sample deep learning detection model}

This paper uses a small sample-based image enhancement method and fine-tunes and merges multiple models of deep learning to further improve the recognition rate of positive image detection.

\subsection{Image recognition convolutional neural network and loss function optimization}

Convolutional neural network is one of the representative algorithms of deep learning, and image recognition based on deep learning also uses convolutional neural network. The convolutional neural network is characterized by its ability to characterize learning and translation invariance. Compared with traditional image recognition algorithms, it has a considerable breakthrough, and its recognition accuracy is greatly improved.

The loss function is a function used to calculate the difference between the label value and the predicted value. It must be used when the deep learning model is compiled. The loss function is a cost function defined on the training set.

The two-category cost function used this time is: 


$$
L(w, b)=-\frac{1}{N} \sum_{i=1}^{N}\left(y^{(i)} \log f\left(x^{(i)}\right)+\left(1-y^{(i)}\right) \log \left(1-f\left(x^{(i)}\right)\right)\right),
$$

where $f(x)$ can be a sigmoid function or other activation functions in deep learning.

The optimizers provided by Keras are BGD, SGD, MBGD, Momentum, NAG, Adagrad, Adadelta, RMSprop, Adam. This experiment mainly uses rmsprop and adam optimizers. The relationship between the loss function and the optimizer balances the detection quality and minimizes the loss of image features.

\subsection{Image enhancement of small samples}

In the past, deep learning must use a large number of training samples as support, otherwise it is prone to overfitting during training. However, this experiment uses the patient's CT image data. Due to the patient's privacy problem, the amount of data is seriously insufficient, so a small sample of image enhancement technology is used to perform a large amount of data expansion. This experiment uses the latest keras image augmentation technology. Compared with the traditional image augmentation technology using pillow or opencv, this technology is simpler and faster. The optional expansion methods basically meet the needs, the generated pictures and the randomness is stronger.

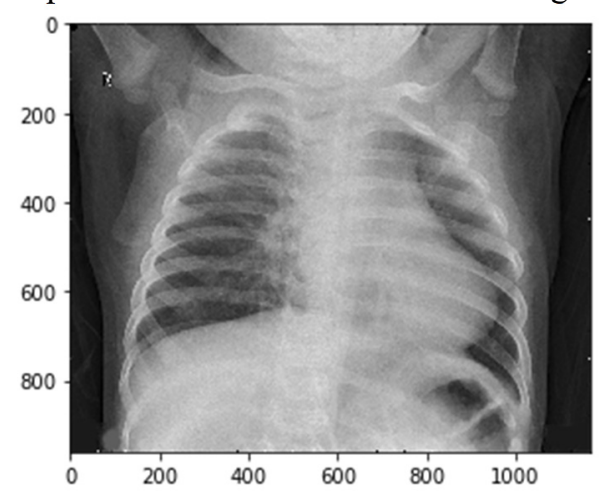

Fig.1. Before extension

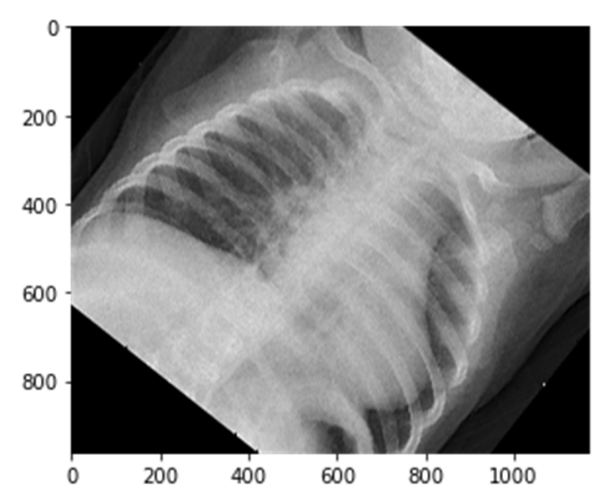

Fig.2. After enlargement

The picture before extension is shown in Figure 1, and the picture after enlargement is shown in Figure 2.

\subsection{Model fusion}

Model fusion is a method of combining a group of base classifiers in a certain way to improve the overall performance of the model. This model fusion is similar to the voting mechanism, that is, multiple model prediction results are voted, and the minority obeys the majority. Finally, the hit probability of the majority is output.

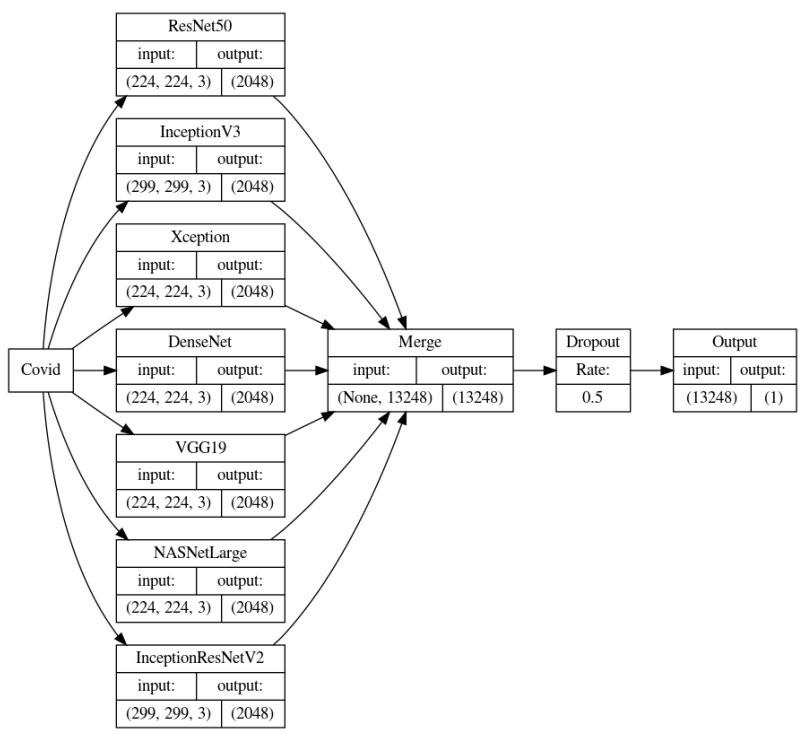

Fig.3. Model fusion diagram

The fusion model of Inception_v3, DenseNet, ResNet50, Xception, VGG19, NASNetLarge, and InceptionResNetV2 is used this time, as shown in Figure 3. These models have their own advantages. The Inception model increases the adaptability of the network to multiple scales. DenseNet improves the flow of information and gradients in the network, and reduces the number of parameters. Resnet proposes a residual structure and a bottleneck layer structure to solve the depth For the degradation problem of the network. Xception proposes a deep separable convolution operation, which reduces a large number of parameters. The VGG19 structure is relatively simple. A simple network can still obtain high accuracy. NASNetLarge uses a neural structure search framework to build Very high accuracy. InceptionResNetV2 is improved from InceptionV3, and its frame accuracy is higher than InceptionV3.

\section{Experimental process}

\subsection{Introduction to Data Set}

The data set of this experiment comes from kaggle and github. The number of samples is 6000 , and the training set is 2450. Data division is shown in Figure 4.

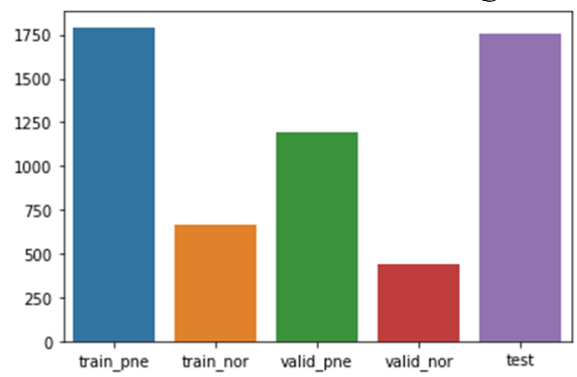

Fig.4. Data set distribution 
Legend: train_pne is the pneumonia training set, train_nor is the non-pneumonia training set, vaild_pne is the pneumonia validation set, vaild nor is the non-pneumonia validation set, and test is the test set.

\subsection{Model Fusion}

The fusion model of Inception_v3, DenseNet, ResNet50, Xception, VGG19, NASNetLarge, and InceptionResNetV2 is used this time, as shown in Figure 5. First download these official models and use these models as base models, and then use the gap layer to directly realize the regularization of the entire network structure and prevent overfitting. Second, two generators are used to generate a large amount of enhanced image data, and then the enhanced image data is used to derive the feature vector of each model. After that, the feature vector is fused through the np.concatenate function, and the model fusion is completed.

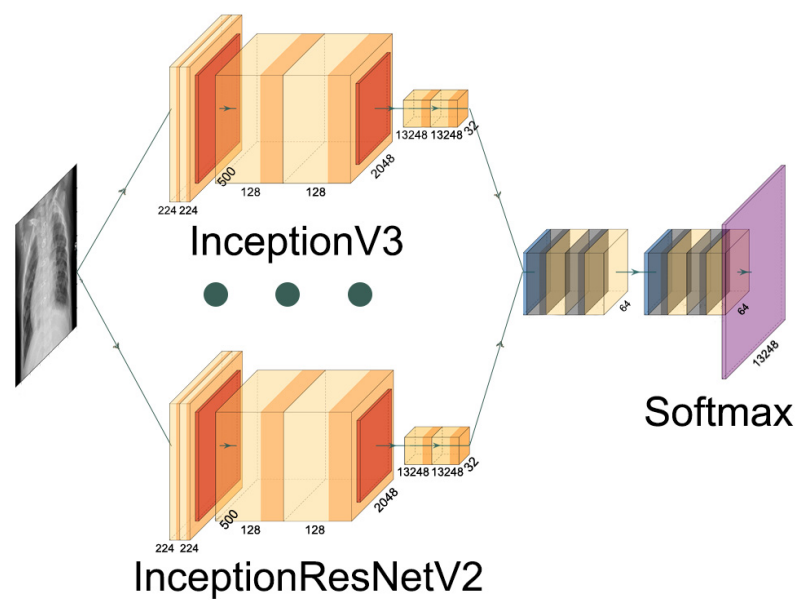

Fig. 5. model fusion diagram

\subsection{Model fine-tuning}

Build a fine-tuning model class to properly fine-tune the final model to improve accuracy. After fine-tuning, dropout is used to randomly select some layers to prevent overfitting, and then perform model training.

\subsection{Machine configuration environment and model training}

This training environment is kaggle, which is essentially a jupyternotebook page built by docker. The GPU used by kaggle is Nvidia Tesla P100-PCIE-16GB $1.3285 \mathrm{GHz}$, the CPU is Intel(R) Xeon(R) CPU 2.3Ghz, and the memory is $14 \mathrm{~GB}$. The disk size is $5.2 \mathrm{~GB}$. The Aconda version is 3.6.6. Machine learning and deep learning modules such as tensorflow, keras, and sklearn are pre-installed. These modules are the latest version.

The final model training optimizer uses Adam, the learning rate is 0.00001 , the loss function is binary_crossentropy, batch_size is 2, epochs is 100, and validation_split is 0.4 .

\subsection{Experimental results}

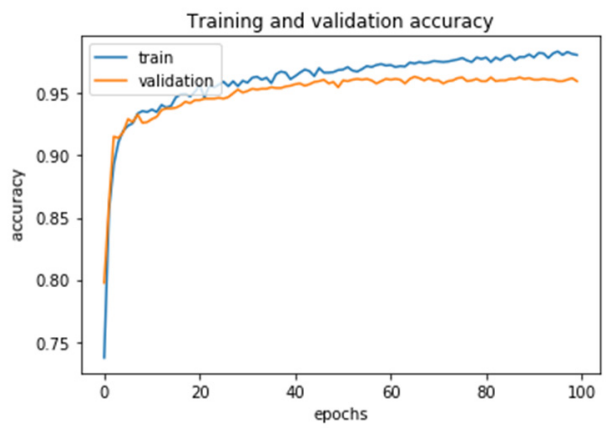

Fig. 6. fusion model accuracy

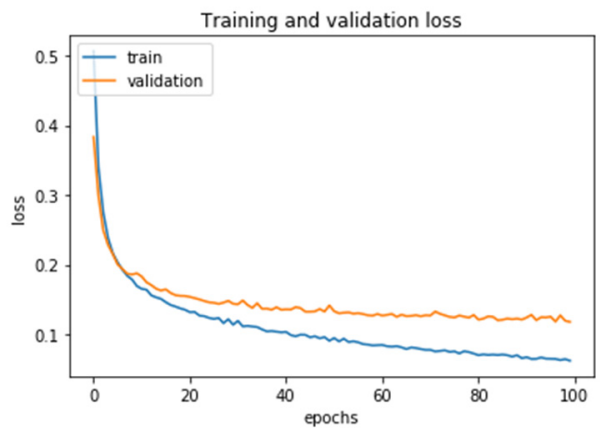

Fig. 7. fusion model loss

According to the final experimental results, the Figure 6 shows accuracy and the Figure7 shows loss, this training has neither over-fitting nor under-fitting. The accuracy of the final model is $96 \%$, which has high reference value in the diagnosis of new coronary pneumonia.

\section{Conclusion}

As the large demand for new coronary pneumonia covid19 image recognition samples, the recognition accuracy is not ideal, this paper designs a new coronary pneumonia positive recognition method based on small samples, and uses image data enhancement to expand a large number of samples, solving the problem of large sample demand. Through a series of operations such as model migration, model fusion, and model fine-tuning, the recognition accuracy is improved. The experimental results show that the recognition accuracy of this experiment is about $96 \%$, which has a high reference value for the diagnosis of new coronary pneumonia, and proves the effectiveness of the method in this paper.

\section{Acknowledgements}

The funding was provided by Natural Science Foundation of Gansu Provincial Department of Science and Technology (NO: 2019A-030). 


\section{References}

1. Novel Coronavirus Pneumonia Tracking[EB/OL]. http://www.bing.com/covid.(2020)

2. National Health Commission of the People's Republie of China [EB/OL]. http://www.nhc.gov.cn.(2020)

3. National Administration of Traditional Chinese Medicine[EB/OL].http://www.satcm.gov.cn.(2020)

4. W. Guan, Z Ni, Y. Hu, W. Liang, C. Ou, J. He, L. Liu, H. Shan, C. Lei, and D. Hui, "Clinical characteristics of coronavirus disease 2019 in China," New England journal of medicine, 382, 1708-1720 (2020)

5. F. Wu, S. Zhao, B. Yu, Y. Chen, W. Wang, Z. Song, Y. Hu, Z. Tao, J. Tian, and Y. Pei, "A new coronavirus associated with human respiratory disease in China," Nature, 579, 265-269 (2020)

6. Ying Song, Shuangjia Zheng, Liang Li, Xiang Zhang, et al.. Deep learning Enables Accurate Diagnosis of Novel Coronavirus (COVID-19) with CT images. medRxiv. (2020)

7. Z. Feng, Q. Yu, S. Yao, and L. Luo, "Early Prediction of Disease Progression in 2019 Novel Coronavirus Pneumonia Patients Outside Wuhan with CT and Clinical Characteristics," medRxiv, (2020)

8. F. Shan, Y. Gao, J. Wang, W. Shi, N. Shi, M. Han, Z. Xue, D. Shen, and Y. Shi, "Lung infection quantification of COVID-19 in CT images with deep learning," arXiv preprint arXiv:2003.04655, (2020)

9. Y. Wang, M. Hu, Q. Li, X. Zhang, G. Zhai, and N. Yao, "Abnormal respiratory patterns classifier may contribute to large-scale screening of people infected with COVID-19 in an accurate and unobtrusive manner," arXiv preprint arXiv:2002.05534, (2020)

10. L. Yan, H. Zhang, Y. Xiao, M. Wang, Y. Guo, C. Sun, X. Tang, L. Jing, S. Li, and M. Zhang, "Prediction of criticality in patients with severe Covid-19 infection using three clinical features: a machine learning-based prognostic model with clinical data in Wuhan," MedRxiv, (2020)

11. S. Wang, B. Kang, J. Ma, and X. Zeng, "A deep learning algorithm using $\mathrm{CT}$ images to screen for Corona Virus Disease (COVID-19)," medRxiv, (2020)

12. C. Zheng, X. Deng, and Q. Fu, "Deep Learning-based Detection for COVID-19 from Chest CT using Weak Label," medRxiv, (2020)

13. A. Abbas, M. Abdelsamea, and M. Gaber, "Classification of COVID-19 in chest X-ray images using DeTraC deep convolutional neural network," medRxiv, (2020) 